\title{
Overspinning a Nearly Extreme Charged Black Hole via a Quantum Tunneling Process
}

\author{
George E. A. Matsas and André R. R. da Silva \\ Instituto de Física Teórica, Universidade Estadual Paulista, Rua Pamplona 145, 01405-900, São Paulo, São Paulo, Brazil
}

(Received 29 May 2007; published 1 November 2007)

\begin{abstract}
We examine a nearly extreme macroscopic Reissner-Nördstrom black hole in the context of semiclassical gravity. The absorption rate associated with the quantum tunneling process of scalar particles whereby this black hole can acquire enough angular momentum to violate the weak cosmic-censorship conjecture is shown to be nonzero.
\end{abstract}

DOI: 10.1103/PhysRevLett.99.181301

PACS numbers: 04.70.Dy, 04.20.Dw, 04.62.+v

The weak cosmic-censorship conjecture (WCCC) plays a fundamental role in black hole physics [1-3]. The validity of the WCCC is a necessary condition to ensure the predictability of the laws of physics [4]. This conjecture asserts that spacetime singularities coming from the complete gravitational collapse of a body must be encompassed by the event horizon of a black hole. We show that quantum effects may challenge the WCCC.

General relativity is the most successful spacetime theory currently available $[1,5]$. It has provided us with much insight on the WCCC. According to the uniqueness theorems [6], all stationary black hole solutions of EinsteinMaxwell equations are uniquely determined by three conserved parameters: the gravitational mass $M$, the electric charge $Q$, and the angular momentum $J$, which satisfy

$$
M^{2} \geq Q^{2}+(J / M)^{2} .
$$

In particular, $M^{2}=Q^{2}+(J / M)^{2}$ characterizes extreme black holes. (See Ref. [7] for a possible way to generate them.) We note that $M^{2}<Q^{2}+(J / M)^{2}$ are associated with naked singularities rather than black holes. (We assume natural units, $c=G=\hbar=1$, unless stated otherwise.) A number of classical results have given full support to the WCCC. Stationary black holes are stable under linear perturbations [8-11] that yield a good testing ground for this conjecture. By analyzing the time development of a thin charged shell with positive proper mass, Boulware concluded that its total collapse does not lead to a naked singularity [12]. Next, Wald considered an extreme black hole and wondered whether or not it would be possible to slightly overcharge and/or overspin it in order to violate the WCCC [13]. Wald shows that when the hole swallows a classical rotating and/or charged test particle, its mass $M$ increases enough to compensate any extra gain of angular momentum and/or charge during the process such that the WCCC is upheld. (See also Ref. [14].) Even though no one has demonstrated the validity of the WCCC in the context of general relativity yet, it is quite possible that this will be achieved sometime in the future [2].

On the other hand, it is well known that when one takes into account quantum effects some classical results associated with black holes can be deeply modified. For in- stance, the classical theory says that the area of a black hole never decreases under any processes, whereas quantum mechanics shows that it will be reduced as a consequence of the black hole radiance [15]. This celebrated phenomenon which dwells on the theory of quantum fields in curved spacetimes $[16,17]$ triggered a broad interest on the issue of black hole thermodynamics (see Refs. [18,19] and references therein). It seems natural to inquire then as to whether quantum mechanics can affect WCCC as well. An investigation in these lines was recently performed by Ford and Roman [20-22] who analyzed the possibility of violating the WCCC by injecting negative energy into an extreme charged black hole, i.e., $Q=M$ and $J=0$. They concluded that the positive energy flux which always follows the negative one would preserve the WCCC.

This Letter revisits the question of whether or not the WCCC remains valid when one includes quantum effects from a different perspective. We consider a nearly extreme macroscopic Reissner-Nördstrom black hole, $Q / M \lesssim 1$, and analyze a quantum tunneling process whereby the black hole can acquire enough angular momentum to overspin, $M^{2}<Q^{2}+(J / M)^{2}$, and therefore violate the WCCC. This is treated in the context of a well-established semiclassical approach, where the low-energy sector of a free massless scalar field is canonically quantized outside the black hole [23,24] (see also Ref. [25]). We stress that we only deal with low-frequency modes in order to minimize backreaction effects. The absorption rate of scalar particles is calculated and used to discuss in what regime the WCCC could be violated by this quantum tunneling process. We eventually comment on the possible limitations of the semiclassical approach and delineate some aspects that should be present after a full quantum gravity analysis is performed.

The line element of a Reissner-Nördstrom black hole can be written in the form [1]

$$
d s^{2}=f(r) d t^{2}-f^{-1}(r) d r^{2}-r^{2}\left(d \theta^{2}+\sin ^{2} \theta d \phi^{2}\right),
$$

where $f(r)=\left(1-r_{+} / r\right)\left(1-r_{-} / r\right) \quad$ and $\quad r_{ \pm}=M \pm$ $\sqrt{M^{2}-\overline{Q^{2}}}$. The outer event horizon is located at $r=r_{+}$. This black hole is assumed to be macroscopic (i.e., $M$ must be much larger than the Planck mass $M_{P}$ ) in order to 
guarantee the applicability of the semiclassical gravity theory.

We analyze now the process in which massless scalar particles are beamed from far away towards the black hole. Because of the existence of an effective scattering potential, low-energy particles, $\omega \approx 0$, are mostly reflected back to infinity. As a matter of fact, the few particles which enter into the hole must quantum mechanically tunnel into it. We will only focus on soft particles because in this case, backreaction effects which are in general present [26] should be significantly dumped by assuming large enough black holes $\left(M \gg \hbar \omega / c^{2}\right)$. In order to maximize the tunneling probability, it is convenient to consider nearly extreme black holes which may overspin by the absorption of a single particle. We also note that in order to disregard any discharge of the hole by electron-positron pair production at the horizon vicinity [20,27] one must assume black holes with mass $M>10^{5} M_{\odot}$, where $M_{\odot}$ is the solar mass. As a by-product of these requirements, the Hawking temperature associated with these holes, which goes like $\sqrt{M^{2}-Q^{2}} / r_{+}^{2}$, is negligible.

The dynamics of a free massless real scalar field $u_{\lambda \omega l m}$ is described by the usual Klein-Gordon equation $\nabla^{\mu} \nabla_{\mu} u_{\lambda \omega l m}=0$. By using the ansatz

$$
u_{\lambda \omega l m}(t, r, \theta, \phi)=\sqrt{\frac{\omega}{\pi} \frac{\psi_{\lambda \omega l}(r)}{r}} Y_{l m}(\theta, \phi) e^{-i \omega t}
$$

with $\omega \geq 0, l \geq 0(l \in \mathbb{N})$, and $m \in[-l, l]$, we reduce the Klein-Gordon equation to a one-dimensional differential equation for $\psi_{\lambda \omega l}$ :

$$
\left\{-f(r) \frac{d}{d r}\left[f(r) \frac{d}{d r}\right]+V_{\mathrm{eff}}(r)\right\} \psi_{\lambda \omega l}(r)=\omega^{2} \psi_{\lambda \omega l}(r),
$$

where

$$
V_{\text {eff }}(r)=f(r)\left[l(l+1) / r^{2}+2 M / r^{3}-2 Q^{2} / r^{4}\right]
$$

is the effective scattering potential (see Fig. 1). Equation (4) possesses two sets of independent solutions associated with purely incoming modes (i) from the whitehole horizon $\mathcal{H}^{-}, \lambda=\rightarrow$ and (ii) from the past null infinity $\mathcal{I}^{-}, \lambda=\leftarrow$.

Thus, the corresponding scalar field operator $\Phi(x)$ can be expanded using the complete set of normal modes as

$$
\Phi(x)=\sum_{\lambda=\rightarrow l=0}^{\leftarrow} \sum_{m=-l}^{l} \int_{0}^{\infty} d \omega\left[a_{\lambda \omega l m} u_{\lambda \omega l m}(x)+\text { H.c. }\right],
$$

where $u_{\lambda \omega l m}(x)$ are orthonormalized according to the Klein-Gordon inner product [16]. With this normalization, the operators $a_{\lambda \omega l m}$ and $a_{\lambda \omega l m}^{\dagger}$ satisfy the usual commutation relations

$$
\left[a_{\lambda \omega l m}, a_{\lambda^{\prime} \omega^{\prime} l^{\prime} m^{\prime}}^{\dagger}\right]=\delta_{\lambda \lambda^{\prime}} \delta_{l l^{\prime}} \delta_{m m^{\prime}} \delta\left(\omega-\omega^{\prime}\right) .
$$

The vacuum state $|0\rangle$ is defined as $a_{\lambda \omega l m}|0\rangle=0|0\rangle$ for every $\lambda, \omega, l$, and $m[28]$.

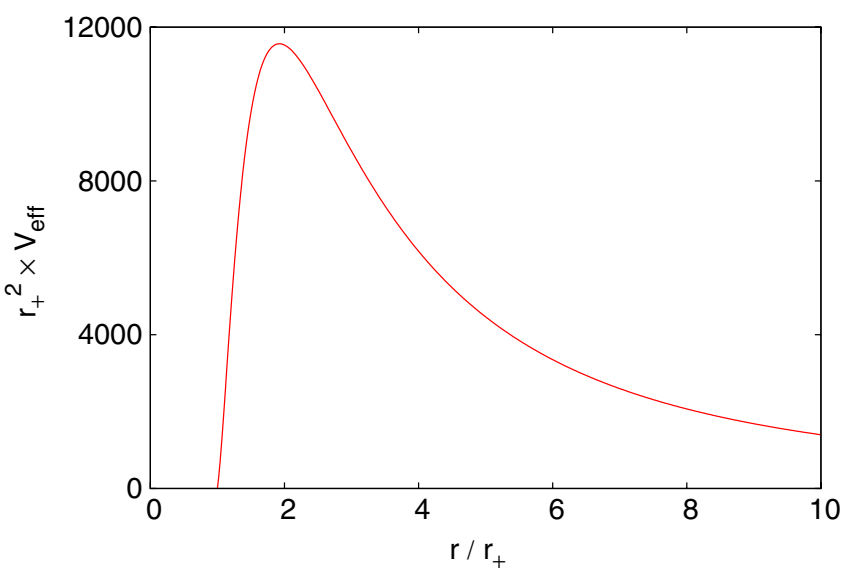

FIG. 1 (color online). The effective scattering potential $V_{\text {eff }}$ is plotted for $M=10^{2} M_{P}, l=413$, and $Q=M-e$, where $e$ is the electron charge. (The numerical values were taken from Fig. 2.)

It is well known that the solutions of Eq. (4) cannot be trivially written in terms of the usual special functions. (For more details, see Ref. [29].) Nevertheless, this is not so in the low-energy regime. In fact, by making $\omega=0$ we can recast Eq. (4) as a Legendre's equation [30]:

$$
\frac{d}{d v}\left[\left(1-v^{2}\right) \frac{d}{d v}\left(\frac{\psi_{\lambda \omega l}(\vec{r}[v])}{\bar{r}[v]}\right)\right]+l(l+1) \frac{\psi_{\lambda \omega l}(\bar{r}[v])}{\bar{r}[v]}=0,
$$

where $v \equiv(2 \bar{r}-1) /\left(\bar{r}_{+}-\bar{r}_{-}\right)$is a dimensionless variable with $\bar{r}=r / 2 M$ and $\bar{r}_{ \pm}=r_{ \pm} / 2 M$. Since we are interested here in particles coming from infinity, we will only write the radial part corresponding to the normal mode $\lambda=\leftarrow$ :

$$
\psi_{\leftarrow \omega l}(\bar{r})=C_{\omega l} \bar{r} P_{l}[v(\bar{r})] .
$$

It is worthwhile to note that $P_{l}[v(\bar{r})]$ behaves as $P_{l}(v) \approx$ $\left[(2 l) ! / 2^{l}(l !)^{2}\right] v^{l}$ for $v \gg 1$ (i.e., $r \gg r_{+}$). Here $C_{\omega l}$ is a normalization constant to be determined. For this purpose, the behavior of the radial mode functions near the horizon and at large distances for any value of $\omega$ is investigated [24]. In lieu of dealing with Eq. (4) we introduce the Regge-Wheeler dimensionless coordinate

$$
r^{*} \equiv \bar{r}+\frac{\bar{r}_{+}^{2} \ln \left|\bar{r}-\bar{r}_{+}\right|-\bar{r}_{-}^{2} \ln \left|\bar{r}-\bar{r}_{-}\right|}{\bar{r}_{+}-\bar{r}_{-}}
$$

to transform it in a Schrödinger like equation:

$$
\left\{-\frac{d^{2}}{d r^{* 2}}+4 M^{2} V_{\text {eff }}\left[r\left(r^{*}\right)\right]\right\} \psi_{\leftarrow \omega l}\left(r^{*}\right)=4 M^{2} \omega^{2} \psi_{\leftarrow \omega l}\left(r^{*}\right) .
$$

At large distances from the event horizon, $r^{*} \gg 1$, the effective scattering potential falls off approximately as $l(l+1) / r^{2}$. This implies that the purely incoming modes from the past null infinity can be written as 


$$
\begin{aligned}
\psi_{\leftarrow \omega l}\left(r^{*}\right) \approx & (-i)^{l+1} M r^{*} h_{l}^{(2)}\left(2 M \omega r^{*}\right) \\
& +i^{l+1} \mathcal{R}_{\omega l} M r^{*} h_{l}^{(1)}\left(2 M \omega r^{*}\right) ; \quad r^{*} \gg 1,
\end{aligned}
$$

where $h_{l}^{(j)}\left(2 M \omega r^{*}\right)$, with $j=1,2$, are the spherical Hankel functions. Next, we recall that near the event horizon, $r^{*}<$ 0 and $\left|r^{*}\right| \gg 1$, the potential barrier is very small: $V_{\text {eff }} \approx 0$. Thus, the purely incoming modes take the form

$$
\psi_{\leftarrow \omega l}\left(r^{*}\right) \approx(2 \omega)^{-1} \mathcal{T}_{\omega l} e^{-2 i M \omega r^{*}} ; \quad r^{*}<0,\left|r^{*}\right| \gg 1 .
$$

Here $\left|\mathcal{R}_{\omega l}\right|^{2}$ and $\left|\mathcal{T}_{\omega l}\right|^{2}$ stand for the reflection and transmission coefficients, respectively, and satisfy the usual probability conservation equation $\left|\mathcal{R}_{\omega l}\right|^{2}+\left|\mathcal{T}_{\omega l}\right|^{2}=1$. The normalization of Eqs. (10) and (11) were accomplished by means of the Klein-Gordon inner product in agreement with the convention fixed below Eq. (6).

In order to determine $C_{\omega l}$ and $\mathcal{T}_{\omega l}$ we need to match the radial functions $\psi_{\leftarrow \omega l}(\bar{r})$ and $\psi_{\leftarrow \omega l}\left(r^{*}\right)$ given in Eqs. (8), (10), and (11), respectively. First, let us write Eq. (10) in the low-energy regime, i.e., $2 M \omega r^{*} \ll 1$ :

$$
\psi_{\leftarrow \omega l}\left(r^{*}\right) \approx \frac{(-i)^{l+1} 2^{2 l+1}(l !) \omega^{l}\left(M r^{*}\right)^{l+1}}{(2 l+1) !} .
$$

We note here that $\mathcal{R}_{\omega l} \approx(-1)^{l+1}+\mathcal{O}(\omega)$. Physically, this means that most incoming particles from the past null infinity will be reflected back by the potential barrier. Now, the asymptotic expansion of Eq. (8) leads to

$$
\psi_{\leftarrow \omega l}(\bar{r}) \approx C_{\omega l} \frac{(2 l) ! \bar{r}^{l+1}}{(l !)^{2}\left(\bar{r}_{+}-\bar{r}_{-}\right)^{l}} .
$$

Then, by comparing Eqs. (12) and (13), one obtains

$$
C_{\omega l}=(-i)^{l+1} \frac{2^{2 l+1}(l !)^{3}\left(\bar{r}_{+}-\bar{r}_{-}\right)^{l} M^{l+1} \omega^{l}}{(2 l) !(2 l+1) !} .
$$

In what follows, $\left|\mathcal{T}_{\omega l}\right|^{2}$ can be determined by fitting Eqs. (11) and (13):

$$
\left|\mathcal{T}_{\omega l}\right|^{2}=\frac{2^{4 l+4}\left(\bar{r}_{+}\right)^{2}\left(\bar{r}_{+}-\bar{r}_{-}\right)^{2 l}(l !)^{6} M^{2 l+2} \omega^{2 l+2}}{[(2 l+1) !(2 l) !]^{2}}
$$

where we recall that $\bar{r}_{ \pm}=r_{ \pm} / 2 M$ and $r_{ \pm}=r_{ \pm}(M, Q)$ is defined below Eq. (2). This is the absorption rate associated with the scalar particles which tunnel the potential barrier towards the hole.

Let us define now the "cosmic-censorship function":

$$
\mathcal{C}(M, Q, J) \equiv M^{2}\left(M^{2}-Q^{2}\right)-J^{2}
$$

[see Eq. (1)]. The initial configuration of our black hole is given by $\mathcal{C}(M, Q, 0)=M^{2}\left(M^{2}-Q^{2}\right)>0$. Now, we are able to determine under what conditions the weak cosmic-censorship conjecture could be violated by the quantum tunneling process. Assuming that a single particle $a_{\leftarrow \omega l m}^{\dagger}|0\rangle$ coming from far away enters into the hole, the value of the cosmic-censorship function becomes

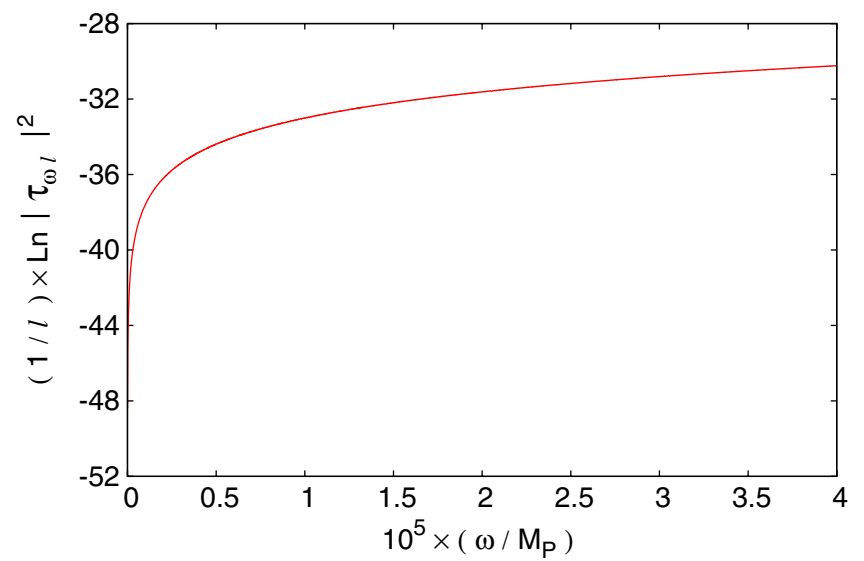

FIG. 2 (color online). The absorption probability $\left|\mathcal{T}_{\omega l}\right|^{2}$ as a function of the frequency $\omega$ is plotted. Here $M=10^{2} M_{P}, Q=$ $M-e$, and $l=413$ which is the smallest angular momentum quantum number whereby the hole is transformed into a naked singularity in this case.

$$
\mathcal{C}(M+\omega, Q, J)=\mathcal{C}(M, Q, 0)-J^{2}+\mathcal{O}(\omega),
$$

where we have required $\omega / M \ll \mathcal{C}(M, Q, 0) / M^{4} \ll 1$. Thus, for particles with small enough frequencies, the cosmic-censorship function will take negative values provided that

$$
l(l+1)>\mathcal{C}(M, Q, 0)
$$

Although black holes near extremity are the ones which require particles with least angular momenta to overspin, $l$ may be very large depending on the black hole mass. For $Q=M-e, \quad$ Eq. (18) becomes $l(l+1)>M^{3}(2 e-$ $e^{2} / M$ ). (See, e.g., Refs. [23,24] for other instances where our semiclassical approach was used with success in the context of low frequencies and large angular momenta.) In Figs. 2 and 3 we plot $\left|\mathcal{T}_{\omega l}\right|^{2}$ as a function of the particle frequency $\omega$. Our semiclassical analysis suggests that

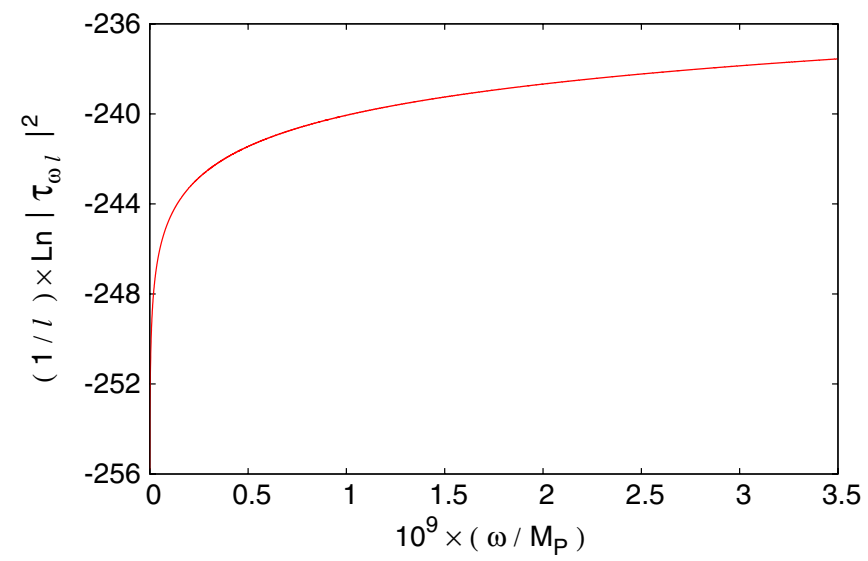

FIG. 3 (color online). The absorption probability $\left|\mathcal{T}_{\omega l}\right|^{2}$ as a function of the frequency $\omega$ is plotted. Here we have chosen $M=10^{5} M_{\odot}$ (no discharge condition), $Q=M-e$, and $l=$ $1141721 \times 10^{58}$. 
WCCC can be quantum mechanically violated. Note that the absorption rate for the black hole mass $M=10^{2} M_{P}$ is much larger than the corresponding one for $M=10^{5} M_{\odot}$ [31]. This can be seen as an indication that perhaps any processes which convert black holes into naked singularities should have a quantum-mechanical nature.

In conclusion, we show how quantum effects can lead to a violation of the weak cosmic-censorship conjecture for nearly extreme charged black holes. It seems to us that a full quantum gravity theory should eventually play a major role in the context of extreme black holes (see also Preskill et al. [32]) either to rescue the WCCC or to unveil the physical nature of the "naked singularities" which we would be forced to face. Nevertheless, we believe that as far as we do not have a complete theory of quantum gravity yet (where backreaction and other possible effects will be fully taken into account), the semiclassical formalism can bring useful new insights and may guide future research on this issue.

G.M. would like to thank J. Castiñeiras, L.C.B. Crispino, A. Higuchi, and D. A. T. Vanzella for profitable discussions. G. M. acknowledges partial support from Conselho Nacional de Desenvolvimento Científico e Tecnológico and Fundação de Amparo à Pesquisa do Estado de São Paulo and A. S. acknowledges full support from Fundação de Amparo à Pesquisa do Estado de São Paulo.

[1] R.M. Wald, General Relativity (The University of Chicago Press, Chicago, 1984).

[2] R. M. Wald, arXiv:gr-qc/9710068.

[3] C.J.S. Clarke, Classical Quantum Gravity 11, 1375 (1994).

[4] S. W. Hawking, Phys. Rev. D 14, 2460 (1976).

[5] S. W. Hawking and G. F. R. Ellis, The Large Structure Scale of Space-Time (Cambridge University Press, Cambridge, England, 1973).

[6] W. Israel, Phys. Rev. 164, 1776 (1967); Commun. Math. Phys. 8, 245 (1968); B. Carter, Phys. Rev. Lett. 26, 331 (1971); S. W. Hawking, Commun. Math. Phys. 25, 152 (1972); D. C. Robinson, Phys. Rev. D 10, 458 (1974); Phys. Rev. Lett. 34, 905 (1975); J. Ipser, Phys. Rev. Lett. 27, 529 (1971).

[7] Ch. J. Farrugia and P. Hajicek, Commun. Math. Phys. 68, 291 (1979).

[8] C. V. Vishveshwara, Phys. Rev. D 1, 2870 (1970).
[9] R. Price, Phys. Rev. D 5, 2419 (1972); 5, 2439 (1972).

[10] B. S. Kay and R. M. Wald, Classical Quantum Gravity 4, 893 (1987).

[11] B. F. Whiting, J. Math. Phys. (N.Y.) 30, 1301 (1989).

[12] D. G. Boulware, Phys. Rev. D 8, 2363 (1973).

[13] R. M. Wald, Ann. Phys. (Leipzig) 82, 548 (1974).

[14] T. Needham, Phys. Rev. D 22, 791 (1980).

[15] S. W. Hawking, Nature (London) 248, 30 (1974); Commun. Math. Phys. 43, 199 (1975).

[16] N.D. Birrel and P.C.W. Davies, Quantum Fields in Curved Space (Cambridge University Press, Cambridge, England, 1982).

[17] S. A. Fulling, Aspects of Quantum Field Theory in Curved Space-Time (Cambridge University Press, Cambridge, England, 1989).

[18] R. M. Wald, Quantum Field Theory in Curved Spacetimes and Black Hole Thermodynamics (University of Chicago Press, Chicago, 1994).

[19] R. M. Wald, Living Rev. Relativity 4, 6 (2001).

[20] L. H. Ford and T. A. Roman, Phys. Rev. D 46, 1328 (1992).

[21] L. H. Ford and T.A. Roman, Phys. Rev. D 41, 3662 (1990).

[22] L. H. Ford, arXiv:gr-qc/0504096.

[23] A. Higuchi, G. E. A. Matsas, and D. Sudarsky, Phys. Rev. D 56, R6071 (1997).

[24] J. Castiñeiras and G.E. A. Matsas, Phys. Rev. D 62, 064001 (2000).

[25] L.C.B. Crispino, A. Higuchi, and G. E. A. Matsas, Classical Quantum Gravity 17, 19 (2000).

[26] P. R. Anderson, W. A. Hiscock, and B. E. Taylor, Phys. Rev. Lett. 85, 2438 (2000); B. E. Taylor, W. A. Hiscock, and P. R. Anderson, Phys. Rev. D 61, 084021 (2000).

[27] G. W. Gibbons, Commun. Math. Phys. 44, 245 (1975).

[28] D. G. Boulware, Phys. Rev. D 11, 1404 (1975); 12, 350 (1975).

[29] P. Candelas, Phys. Rev. D 21, 2185 (1980); B. P. Jensen and P. Candelas, ibid. 33, 1590 (1986); 35, 4041 (1987).

[30] G. B. Arfken and H. J. Weber, Mathematical Methods for Physicists (Academic, New York, 1995).

[31] Modes with large $l$ can be thought of as particles approaching the hole in a highly nonradial way (large impact parameter). Large $l$ values induce a large centrifugal barrier which cannot be overpassed classically by particles with low energies but still can be tunneled quantum mechanically by them. In order to consider lower angular momenta (and, thus, centrifugal barriers), we should take smaller values of $M$.

[32] J. Preskill, P. Schwarz, A. Shapere, S. Trivedi, and F. Wilczek, Mod. Phys. Lett. A 6, 2353 (1991). 